\title{
\% \\ IJCRR \\ Section: Healthcare \\ Portsmouth POSSUM System in General Surgical Practice and Identifying Risk Factors for Low Outcome
}

Sci. Journal Impact

Factor: 6.1 (2018)

ICV: 90.90 (2018)

(c) (7) (8)

Copyright@IJCRR

\author{
R. G. Naniwadekar, Dilip Patil, A. M. Shah, Harshal Bhoi, R. N. Sarwate
}

Department of Surgery, Krishna Institute of Medical Sciences Deemed To Be University, Karad India.

\section{ABSTRACT}

Introduction: The essential point of any surgery is to cause a decrease in grimness and death rates.

Aim: This examination was embraced to evaluate the legitimacy of P-POSSUM scoring framework in patients going through significant medical procedures in our arrangement and, to attempt to dissect the reasons for low result in this high hazard groups is by looking at the effect on the unfriendly result.

Methods: A total of 218 major surgical operations were studied in patients admitted in the general surgery department of Krishna Hospital, Karad.

Results: A study group consisted of 76 elective, 112 emergency and 30 immediate cases. Duodenal perforation (37), Laparotomy is accounted for 175 cases, Resection anastomosis accounted for 27 cases, Amputation accounted for 12 cases, Cholecystectomy with choledochotomy accounted for 2 cases and Embolectomy accounted for 2 cases. 17 patients died among 112 emergency surgeries except for malignancy, Wound infection (56 cases, 25.92\%), accounted for the majority of complications.

Conclusion: This study subsequently approves P-POSSUM as a legitimate method for surveying ampleness of care given to the patient. P-POSSUM can be utilized for careful review to survey and improve the nature of careful consideration and result in better result to the patient.

Key Words: Portsmouth, POSSUM, Surgical, Risk factors, Wound infection, Outcome.

\section{INTRODUCTION}

The essential point of any surgery is to cause a decrease in grimness and death rates... It is by looking at the effect on the unfavourable result; we can survey the proficiency of that specific strategy and evaluate the nature of care gave to the patient. However, correlation utilizing rough dismalness and death rates is erroneous, due to distinction by and large wellbeing of the nearby populace and variable introduction of the patient's condition ${ }^{1,2,3}$.

Hazard scoring tries to evaluate a patient's danger of unfriendly result dependent on the seriousness of ailment got from information accessible at the beginning phase of the medical clinic remain ${ }^{4}$. The conceivable result of a careful activity must be resolved to cause the development of more successful treatment regimens.
Consequently, there is a requirement for an exact danger balanced scoring framework, which ought to be explicit to the patient being considered, should join the impact of the analysis for which he is being oppressed for a medical procedure, regardless of whether elective or crisis and take into consideration evaluation of the variable introduction of every patient, to permit appraisal of the productivity of the specific methodology performed.

It ought to likewise be anything but difficult to utilize, quick and similar among various patient gatherings.

Such a scoring framework would take into consideration the examination of the nature of care gave. it could be utilized to help set a benchmark satisfactory antagonistic result rate for a specific system, by looking at the death rates among various specialists. It would take into account the correlation of viability of different methodology by contrasting the

\section{Corresponding Author:}

Dr. Dilip Patil, Department of Surgery, Krishna Institute of Medical Sciences Deemed To Be University, Karad India. Email: hodsurgery@kimsuniversity.in

ISSN: 2231-2196 (Print)

Received: 22.07 .2020
ISSN: 0975-5241 (Online)

Revised: 20.08 .2020
Accepted: 22.09 .2020
Published: 27.10 .2020 
distinction in saw with expected death rates.it would result in a better and meaningful 'surgical audit' and also help in the faster adaptation of a new procedure by comparing the reduction.

It has held this significance till to-date and if the expressions 'of records' are excluded, it is actually what clinical review means. ${ }^{5}$ Attempts to examine the consequences of medicines have been made since relic however the investigations have frequently been one-sided by reconceptions or flawed rationale. The Royal College of Surgeons of England has characterized the review as the "orderly evaluation of the execution and out happen to any cycle with regards to endorsed targets and guidelines. ${ }^{6}$

The review has three segments - structure, cycle, and result. In 1982, Sheldon characterized the clinical review as 'An investigation of the result of part of the structure, cycle and result of clinical consideration did by those by and by occupied with the movement worried, to gauge whether set targets have been achieved and in this manner survey the nature of care conveyed'. The review of the structure is regulatory. The review of the cycle is that, if right advances are taken in the right request, the result estimated in products or administrations will be agreeable and the review of the result is clear as crystal. $^{7}$

Along these lines review is considerably more than just information assortment, it is reciprocal to investigate, instruction, a promise to progress in care by invigorating further examination, guaranteeing that training is recorded, surveyed and made responsible, subsequently bringing about improved practise propensities. ${ }^{8}$,

A careful review is certainly not another wonder. As right on time as 1750 BC, King Hammurabi of Babylon gave orders for the discipline of careless doctors, especially specialists. In such a declaration found at Susa in Iran and recorded on a 2-m-high dark diorite stone, Hammurabi expresses that: On the off chance that a specialist incurs a genuine injury with his activity blade on a liberated person's slave and murders him, the specialist must supplant the slave with another. If a specialist has treated a liberated individual yet caused a genuine physical issue from which the man bites the dust, or on the off chance that he has opened a boil and the man goes dazzled, the man is to remove his hands. As anyone might expect, interior medication, as opposed to the medical procedure, was famous around then. In reality, to numerous specialists today, this decree despite everything is by all accounts demanded in a sublimated manner.

Many scoring frameworks anticipate the danger of mortality with changing degrees of exactness. Anyway, grimness is generally overlooked. The most popular and most broadly utilized scoring framework is APACHE II (Acute Physiology And Chronic Health Evaluation) which is ideal in the serious consideration tolerant however requires 24 hours of perception and gauging tables for singular sickness states. Additionally, the APACHE III scoring framework has been as of late presented. While all these scoring frameworks are utilized in a commonly debilitated patient, none are solely for careful patients. POSSUM (Physiological and Operative Severity Score for the enUmeration of Mortality and dreariness) is the main scoring framework which is intended for elite use in careful cases.

POSSUM stands for Physiologic and Operative Severity Score for the enUmeration of Mortality and Morbidity and has been proposed as a danger balanced scoring framework to take into consideration direct examination between the watched and expected antagonistic result rates9. It has been called as a specialist based scoring framework. It uses 12 physiologic/biochemical variables, and 6 operative variables to give an estimation of mortality risk.

Anaesthetists are generally less aware (than surgeons) of POSSUM than other scoring systems e.g. ASA for general risk prediction and APACHE for critically ill patients in ICU. ASA is too simplistic and highly subjective and APACHE but requires fewer parameters for its calculation. POSSUM can even be used to give an estimate of risk providing those clinicians handling the data to understand the implications.

P-POSSUM or Portsmouth POSSUM was a modification of the original equation Whiteley et al. that attempted to counteract the overprediction of mortality in low-risk patients. It uses the same variables and may still overpredict mortality in low-risk groups but is a better 'fit' than POSSUM. ${ }^{10,11}$

It has just discovered use all in all, vascular, colorectal, oesophageal and laparoscopic methodology yet the examinations generally included patients in created nations, where the patient attributes, introduction and accessible assets contrast from our setup ${ }^{12 .}$ Consequently, there is a need to test the legitimacy of P-POSSUM scoring framework in the Indian situation where malnourishment is a typical issue, introduction habitually deferred and assets restricted, all of which can impact the patient's difficulty rate, even with satisfactory nature of care gave. Thus, the scoring framework ought to have the option to join these variables to anticipate a precise death rate.

The P-POSSUM scoring framework, which incorporates both physiological and usable discovering boundaries, has been proposed to address these worries. Subsequently, there is have to test whether the P-POSSUM scoring framework can viably address these worries while showing up at the normal death rate in the Indian situation.

Significant medical procedures (elective and crisis), as characterized by the POSSUM scoring framework, establish the significant high hazard gathering of patients where, the ex- 
amination of saw to expected death rate would be required to yield critical outcomes and, assurance of the potential foundations for the unfriendly result in patients who capitulate following the surgery, would be more useful.

This examination was attempted to evaluate the legitimacy of P-POSSUM scoring framework in patients going through significant medical procedures in our arrangement and, to attempt to dissect the reasons for low result in this high hazard gathering.

\section{METHODS}

\section{Source of data}

This prospective study was carried out after ethical clearance from the ethical committee on patients undergoing major general surgical procedures admitted in the department of general surgery of Krishna hospital of KIMS, Karad.

\section{Study period}

The study period was two years and the period of follow up was 30 days following the surgical procedure.

\section{Method of collection of data}

Patients conceded under the broad medical procedure and booked to go through major surgeries were scored by their physiological and employable discoveries utilizing a proforma sheet Patients were educated concerning the points and goals of the study and an itemized educated composed assent was taken before incorporation into the examination. The examination convention was endorsed by the neighbourhood moral leeway advisory group of this clinic.

\section{RESULTS}

A total of 218 major surgical operations were performed between May 2006 to May 2008 which were available for final analysis.

Twenty patients underwent two surgical procedures and five patients underwent $>2$ surgical procedures. There were 142 emergencies, 76 elective procedures and 30 immediate procedures.

Other, represents further indications of laparotomy in our study group- hepatic abscess, spleen abscess, biliary leak, blunt trauma to abdomen causing liver and spleen laceration, Merkel's diverticulum pathology, haemorrhagic pancreatitis, retroperitoneal mass, bull horn injury to the abdomen with the diaphragmatic tear, foreign body impaction in the terminal ileum.
Table 1: Case distribution as per the mode of surgery

\begin{tabular}{lcc} 
Mode of surgery & No. of cases & Percentage \\
Elective & 76 & 34.86 \\
Emergency & 112 & 51.38 \\
Immediate & 30 & 13.76 \\
\hline
\end{tabular}

Table 1 revels that there were 112 (51.38\%) emergency, 76 (34.86\%) elective and $30(13.76 \%)$ immediate surgeries performed.

Table 2: Types of major surgeries performed Table no 2 founds that there were five types of major surgeries performed in our group, represented in table and graph below.

\begin{tabular}{lcc} 
Type of surgery & No. of cases & Percentage \\
Laparotomy & 175 & $80.28 \%$ \\
Resection anastomosis & 27 & $12.39 \%$ \\
Amputation & 12 & $5.50 \%$ \\
$\begin{array}{l}\text { Cholecystectomy with } \\
\text { choledochotomy }\end{array}$ & 2 & $0.92 \%$ \\
Embolectomy & 2 & $0.92 \%$ \\
\hline
\end{tabular}

Table 3: Outcome of surgery

\begin{tabular}{lcc} 
Outcome of surgery & No. of cases & Percentage \\
Alive & 187 & $85.78 \%$ \\
Dead & 31 & $14.22 \%$ \\
\hline
\end{tabular}

Table 3 showed that Out of 218 procedures studied, 31 of them were associated with the death of the patient resulting in a crude mortality rate of $14.22 \%$ represented in the graph.

\begin{tabular}{|c|c|c|c|}
\hline $\begin{array}{l}\text { Age in years } \\
\text { (score) }\end{array}$ & No. of cases & $\begin{array}{c}\text { No. of cases } \\
\text { dead }\end{array}$ & $\begin{array}{c}\text { No of cases } \\
\text { alive }\end{array}$ \\
\hline$<60(1)$ & 170 & $20(11.76 \%)$ & $155(91.18 \%)$ \\
\hline $61-70(2)$ & 28 & $6(21.43 \%)$ & $22(78.57 \%)$ \\
\hline$>71(4)$ & 20 & $5(25 \%)$ & $15(75 \%)$ \\
\hline
\end{tabular}

Table 4 reveals that there were 48 surgeries $(22.02 \%$ of total cases) performed on patients with age more than 60 years and these cases accounted for 11 deaths $(35.48 \%$ of total deaths). The proportion of death increases as the PPOSSUM score for age increases. However this increasing trend was not statistically significant $\left(\chi^{2}=4.478, \mathrm{df}=3\right.$, $\mathrm{p}=0.214$ ). 


\section{Score wise distribution of cases and mortality for blood pressure}

A total of 98 surgeries (44.95\% of total cases) were performed on patients with higher POSSUM score for blood pressure and these cases accounted for 26 deaths $(83.87 \%$ of total deaths). The proportion of death was high in abnormal P-POSSUM score in comparison to the proportion of death in normal P-POSSUM score and was found to be statistically significant. $\left(\chi^{2}=35.296, \mathrm{df}=3, \mathrm{p}=0.000\right)$.

\section{Score wise distribution of cases and mortality for pulse rate}

A total of 163 surgeries $(74.77 \%$ of total cases) were performed on patients with higher POSSUM scores for pulse rate and accounted for 28 deaths (90.32\% of total deaths). The proportion of death significantly increases as P-POSSUM for pulse rate increases and was statistically significant. $\left(\chi^{2}=33.084, d f=3, p=0.000\right)$.

\section{Score wise distribution of cases and mortality for Glasgow coma scale}

There were 10 cases $(4.59 \%$ of total cases) with low Glasgow extreme lethargies scale score, who were exposed to a medical procedure and represented 3 passings ( $9.68 \%$ of total death). There was one patient with a score of less than 9 with no mortality. The proportion of death was high in abnormal P-POSSUM score for GCS in comparison to the proportion of death in normal P-POSSUM score but was not found to be statistically significant. $\left(\chi^{2}=0.651, \mathrm{df}=2, \mathrm{p}=0.722\right)$.

\section{Score wise distribution of cases and mortality for Haemoglobin}

A majority of surgeries were done on patients with abnormalities in haemoglobin levels, 164 cases $(75.23 \%$ of total cases) and these cases accounted for 23 deaths $(74.19 \%$ of total deaths). The proportion of death was high in abnormal P-POSSUM score for haemoglobin in comparison to the proportion of death in normal P-POSSUM score but was not found to be statistically significant. $\left(\chi^{2}=2.706, \mathrm{df}=3\right.$, $\mathrm{p}=0.439$ ).

\section{Score wise distribution of cases and mortality for White blood cell count}

Surgeries done on patients with leucocytosis accounted for 106 cases $(48.62 \%$ of total 218 cases) with 19 deaths (61.29\% of total deaths) occurring in this group. The proportion of death significantly increases as the P-POSSUM score for WBC increases and was found to be statistically significant. $\left(\chi^{2}=6.884, \mathrm{df}=3, \mathrm{p}=0.007\right)$.

\section{Score wise distribution of cases and mortality for Blood urea}

A total of 47 procedures $(21.56 \%$ of total 218 cases $)$ were performed on patients with elevated blood urea levels and these accounted for 18 out of 31 total deaths $(58.06 \%$ of total deaths). The proportion of death significantly increases as the P-POSSUM score for BUN increases and was found to be statistically significant. $\left(\chi^{2}=26.761, d f=3, p=0.000\right)$.

\section{Score wise distribution of cases and mortality for Serum sodium}

Surgeries done on cases with serum sodium abnormalities accounted for 46 cases ( $21.10 \%$ of total 218 cases), with 12 deaths $(38.71 \%$ of total mortality). The proportion of death was high in abnormal P-POSSUM score for serum sodium in comparison to the proportion of death in normal P-POSSUM score and statistically was found to be significant. $\left(\chi^{2}\right.$ $=7.454, \mathrm{df}=3, \mathrm{p}=0.059$ ).

\section{Score wise distribution of cases and mortality for Serum potassium}

Our study group comprised of 39 surgeries $(17.89 \%$ of total 218 cases) performed on patients with some degree of imbalance in serum potassium concentration which accounted for 10 deaths $32.25 \%$ of total (31) deaths.

The proportion of death increases as P-POSSUM score for serum potassium increase in comparison to the proportion of death in normal P-POSSUM score but statistically was not found to be significant. $\left(\chi^{2}=5.542, \mathrm{df}=3, \mathrm{p}=0.136\right)$.

\section{Score wise distribution of cases and mortality for Electrocardiogram findings}

There were 31 cases with electrocardiographic abnormalities who were subjected to major general surgery out of which 3 patients were with scoring 4 accounting 1 (33.33\%of total cases in this group) death and 28 patients were with scoring 8 accounting 7 ( $25 \%$ of total cases in this group) deaths. The proportion of death was high in abnormal P-POSSUM score for ECG in comparison to the proportion of death in normal P-POSSUM score and statistically was found to be significant $\left(\chi^{2}=8.894, \mathrm{df}=3, \mathrm{p}=0.031\right)$

\section{Score wise distribution of cases and mortality for Multiple surgeries}

There were 31 cases in which multiple surgeries performed, in which 26 cases had undergone 2 surgeries and 5 cases had undergone more than 2 surgeries. The proportion of death significantly increases as the P-POSSUM score increases in comparison to the proportion of death in normal P-POSSUM score and was found to be statistically significant $\left(\chi^{2}=8.863\right.$, 2df, $\mathrm{p}=0.012$ ).

\section{Score wise distribution of cases and mortality for Total blood loss}

In our study, we found the majority of cases resulted in 100$500 \mathrm{ml}$ blood loss (134 cases, $61.47 \%$ of total cases), which 
also accounted for the majority of mortalities (14 cases, $10.45 \%$ of total cases in this group). There were $14(6.42 \%$ of total cases) cases with $500-100 \mathrm{ml}$ blood loss of which $4(28.57 \%$ of total cases in this group) cases died during the study period there were $11(5.05 \%$ of total cases) cases with $>1000 \mathrm{ml}$ blood loss of which $5(45.45 \%$ of total cases in this group) cases died during the study period. The proportion of death increases as P-POSSUM Score for TBL increases, suggesting a correlation of higher blood loss with more adverse outcome and was found to be statistically significant $\left(\chi^{2}=13.645,4 d f \mathrm{p}=0.009\right)$.

\section{Score wise distribution of cases and mortality for Peritoneal contamination}

In a total of 150 surgeries, some degree of peritoneal contamination was found and 75 surgeries were associated with free bowel content, blood or gross pus accounting for 18 (24\%of total cases in this group) deaths, 12 surgeries were associated with localized peritoneal pus with no $(0 \%)$ mortality, 63 surgeries were associated with minor serous peritoneal contamination accounting for $9(14.29 \%$ of total cases in this group) deaths. The proportion of death is high in abnormal P-POSSUM score in comparison to the proportion of death in normal P-POSSUM score, suggesting the association of degree of peritoneal contamination with adverse outcome and was found to be statistically significant. $\left(\chi^{2}=12.196\right.$, $\mathrm{df}=3, \mathrm{p}=0.007$.

\section{Score wise distribution of cases and mortality for Malignancy}

There were 49 patients with malignancies who had undergone surgery. There were 25 cases with primary only, with 2 deaths( $8 \%$ of total cases in this group), 15 cases with lymph node involvement with 1 death $(6.67 \%$ of total cases in this group) and 9 cases with disseminated metastases with no deaths.

There was no increase in the proportion of death as P-POSSUM score increases and statistically also was not significant. $\left(\chi^{2}=3.890, \mathrm{df}=3, \mathrm{p}=0.274\right)$.

\section{Score wise distribution of cases and mortality Mode of surgery}

Out of 76 elective surgeries, there were 4 (5.26\% of total cases in this group) deaths, in 112 emergency surgeries there were 17 (15.18\% of total cases in this group) and in $30 \mathrm{im}-$ mediate surgeries, there were $10(33.33 \%$ of total cases in this group) deaths. The proportion of death significantly increases as the P-POSSUM score for the mode of surgery increases and was found to be statistically significant. $\left(\chi^{2}=14.987, \mathrm{df}=2, \mathrm{p}=0.001\right)$.

\section{DISCUSSION}

In our investigation, we evaluated the legitimacy of P-POSSUM in 218 significant general medical procedures by contrasting the watched death rate and expected death rate. 31 patients died, with mortality rates of $5.26 \%$ among elective and $15.18 \%$ among emergency, $33.33 \%$ among immediate (mean \% of emergency+immediate mortality was $24.255 \%$ ), the total crude mortality rate is $14.22 \%$. Tekkis and others got comparative outcomes (elective $=3.9 \%$, crisis $25 \%$ and generally speaking death pace of $11.1 \%) 13$. Anyway, on utilizing P-POSSUM the normal death rate was 39 passings. On investigation, there was discovered to be no measurably noteworthy distinction between the watched and expected death rates $\left(\chi^{2}=-15.369\right.$, d.f $=9$., $\left.p<0.05\right)$. An O: E ratio of 0.79 was obtained. Similar findings were obtained by Mohilet al ${ }^{14}\left(\mathrm{O}: \mathrm{E}=0.66, \chi^{2}=5.33\right.$, 9 d.f., $\left.\mathrm{p}=0.619\right)$, Tekkis et al. ${ }^{13}$ (O: $\left.E=0.98\right)$, Hence P-POSSUM was able to accurately predict the adverse outcome following major surgery in our study.

On analysing the risk factors we found the proportion of death increases with abnormal P-POSSUM score for all the risk factors studied except for malignancy this could be due to less number of cases causing limitations for study, but it was found to be statistically significant. Wound infection (56 cases, $25.92 \%$ ) and chest infections (30 cases, $15.96 \%$ ) and septicaemia (27 cases, 14.36\%) accounted for the majority of complications. Similar results were obtained by Mohil et al. ${ }^{14}(35 \%, 20 \%)$ respectively for wound infection and chest infection.

\section{CONCLUSION}

This investigation accordingly approves P-POSSUM as a legitimate method for surveying ampleness of care given to the patient. P-POSSUM can be utilized for careful review to survey and improve the nature of careful consideration and result in better result to the patient.

\section{ACKNOWLEDGEMENT}

Authors acknowledge the immense help received from the scholars whose articles are cited and included in references to this manuscript. The authors are also grateful to authors/ editors/publishers of all those articles, journals and books from where the literature for this article has been reviewed and discussed."

Conflict of interest: Nothing to report.

Source of funding: Self-funding. 


\section{REFERENCES}

1. Sagar PM, Hartley MN, Mancey-Jones B, Sedman PC, May J, Macfie J. Comparative audit of colorectal resection with the POSSUM scoring system. British journal of surgery. 1994 Oct;81(10):1492-4.

2. Copeland GP, Jones D, Wilcox A, Harris PL. Comparative vascular audit using the POSSUM scoring system. Annals of the Royal College of Surgeons of England. 1993 May;75(3):175.

3. Murray GD, Hayes C, Fowler S, Dunn DC. Presentation of comparative audit data. British journal of surgery. 1995 Mar;82(3):329-32.

4. Jones HJ, Cossart LD. Risk scoring in surgical patients. British Journal of Surgery. 1999 Feb 1;86(2):149-57.

5. Pawar S, and Shinde, MB. Nursing audit of clinical learning environment among nursing student. International Journal of Advanced Science and Technology, 2020; 29(3), 470-475.

6. Wright JE. The history of the surgical audit. Journal of Quality in Clinical Practice. 1995 Jun 1;15(2):81-8.

7. Varghese AC, Shinde MB, and Gautam A.The incidence and risk factors for ventilator-associated pneumonia (VAP) in the critical care unit. International Journal of Advanced Science and Technology, 2020; 29(4): 1183-1187.
8. Davies MG, Shine MF, Lennon F. Surgical audit under scrutiny. Irish Journal of medical science. 1991 Oct 1;160(10):299.

9. Copeland GP. Comparative audit: fact versus fantasy. British journal of surgery. 1993;80(11):1424-5.

10. Whiteley MS, Prytherch DR, Higgins B, Weaver PC, Prout WG. An evaluation of the POSSUM surgical scoring system. British journal of surgery. 1996;83(6):812-5.

11. Prytherch DR, Whiteley MS, Higgins B, Weaver PC, Prout WG, Powell SJ. POSSUM and Portsmouth POSSUM for predicting mortality. British Journal of Surgery. 1998;85(9):1217-20..

12. Yii MK, Ng KJ. Risk-adjusted surgical audit with the POSSUM scoring system in a developing country. British Journal of surgery. 2002 Jan 1;89(1):110-3.

13. Tekkis PP, Kocher HM, Bentley AJ, Cullen PT, South LM, Trotter GA, Ellul JP. Operative mortality rates among surgeons. Diseases of the colon \& rectum. 2000 Nov 1;43(11):1528-32.

14. Mohil RS, Bhatnagar D, Bahadur L, Dev DK, Magan M. POSSUM and P-POSSUM for the risk-adjusted audit of patients undergoing emergency laparotomy. British journal of surgery. 2004 Apr 1;91(4):500-3. 